\title{
A CONJECTURE OF S. CHOWLA VIA THE GENERALIZED RIEMANN HYPOTHESIS
}

\author{
R. A. MOLLIN AND H. C. WILliAMS \\ (Communicated by Larry J. Goldstein)
}

\begin{abstract}
S. Chowla conjectured that if $p=m^{2}+1$ is prime and $m>26$, then $h_{K}$, the class number of $K=Q(\sqrt{p})$, is greater than 1 . We prove this conjecture under the assumption of the Riemann hypothesis for $\zeta_{K}$, the zeta function of $K$, i.e. the generalized Riemann hypothesis (GRH).
\end{abstract}

It is the purpose of this note to prove the following result.

THEOREM. Let $K=Q(\sqrt{p})$, where $p=m^{2}+1$ is prime and $m>26$. If the Riemann hypothesis holds for $\varsigma_{K}$ then $h_{K}>1$.

Without the GRH hypothesis, this is known as the Chowla conjecture given in [1]. We note that it is an easy consequence of the celebrated Brauer-Siegel theorem. that there are only finitely many such $p$ for which $h_{K}=1$. In [3] Mollin reduced the problem to the case where $m=2 r$ and $r>13$ is prime.

In what follows we make use of an idea of Cornell and Washington [2] to show how to use the GRH to get an effective bound $\left(p>10^{23}\right)$ for which the Chowla conjecture holds. The remaining finite cases are then handled by a simple sieve process. Throughout the remainder of the paper $p$ will denote a prime of the form $4 r^{2}+1$ where $r$ is an odd prime, and $K$ will denote $Q(\sqrt{p})$.

The following result contains facts which are either well known or trivial. Therefore we state it without proof.

LEMMA. Let $y$ be a real number.

(1) If $y>1$, then $\sum 1 / q<\log y$, where the sum ranges over all primes $q \leq y$.

(2) If $y>0$, then $\sum\left(1 / q^{2}\right)<1 / y$, where the sum ranges over all primes $q>y$,

(3) If $|y| \leq 1 / 2$, then $|\log (1-y)+y|<y^{2}$.

Now we are in a position to prove the Theorem.

Let $\varepsilon(q)=(p / q)$ denote the Kronecker symbol where $q$ is a prime. Set

$$
T_{1}(y)=\sum_{q \leq y}[q /(q-\varepsilon(q))] \quad \text { and } \quad T_{2}(y)=\prod_{q>y}[q /(q-\varepsilon(q))] .
$$

Received by the editors August 13, 1986 and, in revised form, December 8, 1986.

1980 Mathematics Subject Classification (1985 Revision). Primary 12A50, 12A25; Secondary $10 \mathrm{~B} 05$.

Key words and phrases. Class number 1, real quadratic field, Riemann hypothesis.

The first author's research is supported by N.S.E.R.C. Canada, Grant \#A8484.

The second author's research is supported by N.S.E.R.C. Canada, Grant \#A7649. 
Therefore $L(1, \chi)=T_{1}(y) T_{2}(y)$, the Euler product for the Dirichlet $L$-function. Clearly we have that $T_{1}(y) \geq \prod_{q \leq y}(q /(q+1))$. Therefore

$$
\log T_{1}(y) \geq-\sum_{q \leq y} \log (q+1 / q)>-\sum_{q \leq y} 1 / q>-\log y
$$

where the last inequality is from Lemma (1). Furthermore, we have from Lemma, (2)-(3), that

$$
\log T_{2}(y)=-\sum_{q>y} \log (1-(\varepsilon(q) / q))>\sum_{q>y} \varepsilon(q) / q-1 / y .
$$

By similar reasoning to that used by Cornell and Washington [2, p. 265] (where the GRH is assumed) we get

$$
\sum_{q>y} \varepsilon(q) / q \geq-B(y)[(4+3 \log y) / \sqrt{y}],
$$

where $B(y)=(\log p)\left((1 / \pi \log y)+\left(5.3 /(\log y)^{2}\right)\right)+4 / \log y+1 / \pi$. Now, from (ii) and (iii), we get

$$
\log T_{2}(y)>-B(y)[(4+3 \log y) / \sqrt{p}]-1 / y .
$$

Hence from (i) and (iv) we have

$$
L(1, \chi)=T_{1}(y) T_{2}(y)>(1 / y) \exp [-B(y)[(4+3 \log y) / \sqrt{p}]-1 / y]
$$

Set $y=(\log p)^{2} ;$ whence $\log y=2 \log \log p$. Thus

$$
\begin{aligned}
& -B(y)[(4+3 \log y) / \sqrt{y}]-1 / y \\
& =-\left\{2 /(\pi \log \log p)+15 /(\log \log p)^{2}+8 /(\log \log p) \log p+4 /(\pi \log p)\right. \\
& \left.\quad+3 / \pi+135 /(2 \log \log p)+12 / \log p+6 \log \log p /(\pi \log p)+1 /(\log p)^{2}\right\} \\
& =d(p),
\end{aligned}
$$

say. Hence $L(1, \chi)>e^{d(p)} /(\log p)^{2}$. However, $2 h_{K} R=\sqrt{p} L(1, \chi)$, where $R=$ $\log (2 r+\sqrt{p})<\log 2 \sqrt{p}$ is the regulator of $K$; whence

$$
h_{K}>\frac{\sqrt{p} e^{d(p)}}{2(\log p)^{2}(\log 2 \sqrt{p})}=F(p),
$$

say. Moreover $-d(p)$ is a decreasing function of $p$ so then $e^{d(p)}$ is an increasing function of $p$, forcing $F(p)$ to be an increasing function of $p$. Thus, if $p>10^{13}$ then $d(p)<4.68, e^{d(p)}>0.009279493$ and $\sqrt{p} /\left(2(\log p)^{2}(\log 2 \sqrt{p})\right) \geq 112.6838154$. Hence $F(p)>1$ for $p>10^{13}$.

Now, to deal with the primes $p<10^{13}$ where $p=4 r^{2}+1$ we note that $r<$ $10^{6 \cdot 5} / 2 \approx 1.6 \times 10^{6}$. Hereafter, we use the fact proved by Mollin in [3] that $h_{K}=1$ is tantamount to all primes less than $r$ being inert in $K$. Select some positive integer $k$ and the first $k$ primes $\left\{q_{i}\right\}_{i=1}^{k}$ with $q_{1}=5$. For each of these $q_{i}$ find (by trial) and tabulate those $S_{i j}$ such that $0 \leq S_{i j} \leq q_{i}-1$ and $\left(\left(4 S_{i j}^{2}+1\right) / q_{i}\right) \neq-1$ where $(/)$ is the Legendre symbol. There are approximately $q_{i} / 2$ of these. If any $r \equiv S_{i j}\left(\bmod q_{i}\right)$ and $r>q_{i}$ then delete this value of $r$ since either: $4 r^{2}+1 \equiv 0$ $\left(\bmod q_{i}\right)$, which means that $4 r^{2}+1$ is not prime, or $\left(\left(4 r^{2}+1\right) / q_{i}\right)=1$ and $q_{i}<r$, which means that $h_{K}>1$. Since half of the $r$ 's are eliminated for each $q_{i}$, then a 
value of $k$ such that $2^{k}>1.6 \times 10^{6}$ should suffice to complete the task. We used $k=100$ and a Fortran program to sieve out as many values of $r<1.6 \times 10^{6}$ as possible. In a matter of a few minutes we found that if $r>13$ and $p=4 r^{2}+1$ is a prime $\left(p<10^{13}\right)$ there exists some $q_{i}<r$ such that $q_{i}$ is quadratic residue of $p$. This proves the Theorem. Q.E.D.

\section{REFERENCES}

1. S. Chowla and J. Friedlander, Class numbers and quadratic residues, Glasgow Math. J. 17 (1976), 47-52.

2. G. Cornell and L. C. Washington, Class numbers of cyclotomic fields, J. Number Theory 21 (1985), 260-274.

3. R. Mollin, Necessary and sufficient conditions for the class number of a real quadratic field to be one and a conjecture of S. Chowla, Proc. Amer. Math. Soc. 102 (1988), 17-21.

Department of Mathematics and Statistics, The University of Calgary, 2500 University Drive N.W., Calgary, Alberta, Canada T2N 1N4

COMPUTER SCIENCE DEPARTMENT, The UNIVERSity OF MANitoba, WinNipeg, MANITOBA, CANADA R3T 2N2 